\title{
International Consensus Statement for the Screening, Diagnosis, and Treatment of Adolescents with Concurrent Attention-Deficit/Hyperactivity Disorder and Substance Use Disorder
}

Heval Özgen ${ }^{a}, \xi$ Renske Spijkerman ${ }^{a}$ Moritz Noack ${ }^{b}$ Martin Holtmann ${ }^{b}$ Arnt S.A. Schellekens ${ }^{c, d}$ Geurt van de Glind $^{\text {d, e }}$ Tobias Banaschewskif ${ }^{f}$ Csaba Bartag, h Alex Begeman ${ }^{\mathrm{i}}$ Miguel Casas ${ }^{\mathrm{j}}$ Cleo L. Crunelle ${ }^{k}$ Constanza Daigre Blanco ${ }^{1-n}$ Søren Dalsgaard ${ }^{\circ}$ Zsolt Demetrovics $^{p}$ Jacomine den Boer ${ }^{i}$ Geert Dom ${ }^{q}$ Valsamma Eapen ${ }^{r}$ Stephen V. Faraone ${ }^{s}$ Johan Franck ${ }^{t}$ Rafael A. González ${ }^{u, v}$ Lara Grau-López ${ }^{I-n, T}$ Annabeth P. Groenman ${ }^{\text {w, } x}$ Malin Hemphälä ${ }^{t}$ Romain Icick ${ }^{y, z}, A$ Brian Johnson ${ }^{s}$ Michael Kaess ${ }^{B, C}$ Máté Kapitány-Fövény ${ }^{D, E}$ John G. Kasinathan ${ }^{F}$ Sharlene S. Kaye ${ }^{G}$ Falk Kiefer $^{H}$ Maija Konstenius ${ }^{t}$ Frances R. Levin' Mathias Luderer ${ }^{J}$ Giovanni Martinotti ${ }^{K}$ Frieda I.A. Matthys ${ }^{L}$ Gergely Meszaros ${ }^{M}$ Franz Moggi $^{N}$ Ashmita P. Munasur-Naidoo ${ }^{\mathrm{O}, \mathrm{P}}$ Marianne Post ${ }^{\mathrm{Q}}$ Sharon Rabinovitz ${ }^{\mathrm{R}}$ J. Antoni Ramos-Quiroga ${ }^{m, n}, S, T$ Regina SalaU Abu Shafi ${ }^{V}$ Ortal SlobodinW Wouter G. Staal ${ }^{X, Y}$ Rainer Thomasius $^{Z}$ Ilse Truter ${ }^{\alpha}$ Michiel W. van Kernebeek ${ }^{\beta}$ Maria C. Velez-Pastrana ${ }^{Y}$ Sabine Vollstädt-Klein ${ }^{H} \quad$ Florence Vorspan ${ }^{z, \delta, \varepsilon, \zeta}$ Jesse T. Young ${ }^{\theta, \eta, 1, k} \quad$ Amy Yule $^{\lambda}$ Wim van den Brink ${ }^{\mathrm{e}, \mu}$ Vincent Hendriks ${ }^{\mathrm{a}, \xi}$

aParnassia Addiction Research Centre (PARC), Parnassia Psychiatric Institute, The Hague, The Netherlands; ${ }^{b}$ Department of Child and Adolescent Psychiatry, LWL-University Hospital, Ruhr-University Bochum, Hamm, Germany; ' Department of Psychiatry, Donders institute, RadboudUMC, Nijmegen, The Netherlands; ${ }^{\text {NNijmegen }}$ Institute for Scientist-Practitioners in Addiction (NISPA), RadboudUMC, Nijmegen, The Netherlands; eInternational Collaboration on ADHD and Substance Abuse (ICASA) Foundation, Nijmegen, The Netherlands; ${ }^{\text {fDepartment }}$ of Child and Adolescent Psychiatry, Central Institute of Mental Health Mannheim (CIMH), Heidelberg University, Mannheim,

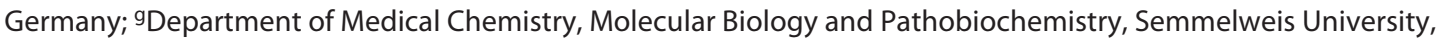

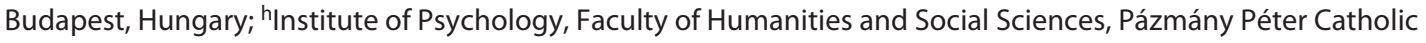
University, Budapest, Hungary; 'De Hoop GGZ, Dordrecht, The Netherlands; 'Department of Psychiatry and Legal Medicine, Autonomous University of Barcelona, Barcelona, Spain; ${ }^{k}$ Department of Psychiatry, University Hospital Brussels, Brussels, Belgium; 'Department of Psychiatry, Mental Health and Addictions, Addiction and Dual Diagnosis Section, Hospital Universitari Vall d'Hebron, Barcelona, Spain; ${ }^{\mathrm{m}}$ Group of Psychiatry, Mental Health and Addictions, Vall d'Hebron Research Institute (VHIR), Barcelona, Spain; 'Biomedical Network Research Centre on Mental Health (CIBERSAM), Barcelona, Spain; ${ }^{\circ}$ Department of Economics and Business Economics, Aarhus University, Aarhus, Denmark; PInstitute of Psychology, ELTE Eötvös Loránd University, Budapest, Hungary; ${ }^{9}$ Collaborative Antwerp

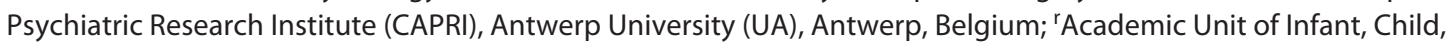
Adolescent Psychiatry South West Sydney, University of New South Wales, Sydney, NSW, Australia; 'separtment of Psychiatry, SUNY Upstate Medical University, Syracuse, NY, USA; ${ }^{t}$ Child- and Adolescent Department, Piteå Hospital, Region Norrbotten, Piteå, Sweden;

Heval Özgen, Renske Spijkerman, Wim van den Brink and Vincent Hendriks contributed equally to this paper.

karger@karger.com www.karger.com/ear

(c) 2020 S. Karger AG, Basel

Karger!
Dr. Heval Özgen

Parnassia Addiction Research Centre (PARC)

Parnassia Psychiatric Institute

Zoutkeetsingel 40, NL-2512 HN The Hague (The Netherlands)

m.h.ozgen@parnassiagroep.nl 
${ }^{u}$ Centre for Mental Health, Division of Brain Sciences, Department of Medicine, Imperial College London, London, UK; 'East London NHS Foundation Trust, Child and Adolescent Mental Health Service (CAMHS) - ADHD clinic,

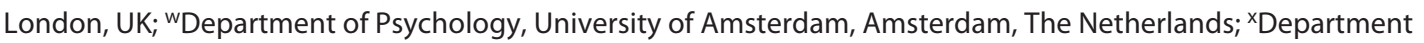
of Child and Adolescent Psychiatry, University of Groningen, University Medical Center Groningen, Groningen, The Netherlands; ${ }^{y}$ Assistance Publique-Hôpitaux de Paris (AP-HP), Groupe Hospitalier Saint-Louis - Lariboisière Fernand Widal, Paris, France; 'ZINSERM U1144, Paris, France; A Inserm UMR-S1144, Université de Paris, Paris, France; BUniversity Hospital of Child and Adolescent Psychiatry and Psychotherapy, University of Bern, Bern, Switzerland; ${ }^{C}$ Department of Child and Adolescent Psychiatry, Center for Psychosocial Medicine, University Hospital Heidelberg, Heidelberg, Germany; DFaculty of Health Sciences, Semmelweis University, Budapest, Hungary; EDrug Outpatient Centre, Nyírő Gyula National Institute of Psychiatry and Addictions, Budapest, Hungary; ${ }^{F}$ Adolescent Mental Health, Justice Health and Forensic Mental Health Network, Sydney, NSW, Australia; ${ }^{G}$ National Drug and Alcohol Research Centre, University of New South Wales, Sydney, NSW, Australia; ${ }^{H}$ Department of Addictive Behaviour and Addiction Medicine, Central Institute of Mental Health, Mannheim, Germany; 'Department of Psychiatry, Columbia University, New York State Psychiatric Institute, New York, NY, USA; 'Department of Psychiatry, Psychosomatic Medicine and Psychotherapy, University Hospital, Goethe University, Frankfurt, Germany; ${ }^{\mathrm{K}}$ Department of Neuroscience, Imaging, Clinical Sciences, University "G.d'Annunzio", Chieti-Pescara, Chieti, Italy; 'Department of Psychiatry, UZ Brussel, Vrije Universiteit Brussel (VUB), Brussels, Belgium; MDepartment of Psychiatry and Psychotherapy, Faculty of Medicine, Semmelweis University, Budapest, Hungary; NUniversity Hospital of Psychiatry, University of Bern, Bern, Switzerland; ${ }^{\circ}$ Department of Pharmacy, Nelson Mandela University, Port Elizabeth, South Africa; ${ }^{P}$ Cipla Medpro Pharmaceuticals, Durban, South Africa; ${ }^{Q}$ Brijder Youth Addiction Treatment, Parnassia Psychiatric Institute, The Hague, The Netherlands; ${ }^{\mathrm{R}} \mathrm{S}$ chool of Criminology and The Unit for Excellence in Research \& Study of Addiction (ERSA), The Center for Rehabilitation Research, University of Haifa, Haifa, Israel; ${ }^{5}$ Department of Psychiatry, Hospital Universitari Vall d'Hebron, Barcelona, Spain; 'Department of Psychiatry and Forensic Medicine, Universitat Autònoma de Barcelona, Barcelona, Spain; ${ }^{U}$ Centre for Psychiatry, Wolfson Institute, Barts \& The London School of Medicine \& Dentistry, Queen Mary University of London, London, UK; VEast London NHS Foundation Trust, London, UK; ${ }^{W}$ Department of Education, Ben-Gurion University, Beer-Sheva, Beer-Sheva, Israel; ${ }^{X}$ Department of Psychiatry, Radboud University Medical Centre, Nijmegen, The Netherlands; ${ }^{Y}$ Karakter Child and Adolescent Psychiatry University Centre, Nijmegen, The Netherlands; ${ }^{\mathrm{Z}}$ German Centre for Addiction Research in Childhood and Adolescence, University Medical Centre Hamburg-Eppendorf, Hamburg, Germany; ${ }^{a}$ Drug Utilization Research Unit (DURU), Department of Pharmacy, Nelson Mandela University, Port Elizabeth, South Africa; ${ }^{\beta}$ Department of Psychiatry, Universitair Ziekenhuis Brussel (UZ Brussel), Vrije Universiteit Brussel (VUB), Brussels, Belgium; ${ }^{\gamma} \mathrm{PhD}$ Program in Clinical Psychology, Universidad Carlos Albizu, San Juan, Puerto Rico; ${ }^{\delta}$ Département de Psychiatrie et de Médecine Addictologique, Hôpital Fernand Widal, Paris, France; ${ }^{\varepsilon}$ Faculté de Médecine, Université de Paris, Paris, France; ${ }^{\zeta}$ FHU NOR-SUD Network of Research in Substance Use Disorders, Paris, France; ${ }^{\ominus}$ Melbourne School of Population and Global Health, The University of Melbourne, Melbourne, VIC, Australia; 'Murdoch Children's Research Institute, Melbourne, VIC, Australia; 'School of Population and Global Health, The University of Western Australia, Perth, WA, Australia; ${ }^{K}$ National Drug Research Institute, Curtin University, Perth, WA, Australia; ${ }^{\lambda}$ Boston Medical Center, Boston University School of Medicine, Boston, MA, USA; ${ }^{\mu}$ Amsterdam University Medical Centers, location Academic Medical Center, Amsterdam, The Netherlands; ${ }^{\complement}$ Curium, Department of Child and Adolescent Psychiatry, Leiden University Medical Center, Leiden, The Netherlands

\section{Keywords}

Consensus statement · Attention-deficit/hyperactivity

disorder · Substance use disorder · Adolescents

\section{Abstract \\ Background: Childhood attention-deficit/hyperactivity disorder (ADHD) is a risk factor for substance misuse and substance use disorder (SUD) in adolescence and (early) adulthood. ADHD and SUD also frequently co-occur in treatment-seeking adolescents, which complicates diagno-}

sis and treatment and is associated with poor treatment outcomes. Research on the effect of treatment of childhood ADHD on the prevention of adolescent SUD is inconclusive, and studies on the diagnosis and treatment of adolescents with ADHD and SUD are scarce. Thus, the available evidence is generally not sufficient to justify robust treatment recommendations. Objective: The aim of the study was to obtain a consensus statement based on a combination of scientific data and clinical experience. Method: A modified Delphi study to reach consensus based upon the combination of scientific data and clinical experience with a multidisci- 
plinary group of 55 experts from 17 countries. The experts were asked to rate a set of statements on the effect of treatment of childhood ADHD on adolescent SUD and on the screening, diagnosis, and treatment of adolescents with comorbid ADHD and SUD. Results: After 3 iterative rounds of rating and adapting 37 statements, consensus was reached on 36 of these statements representing 6 domains: general $(n=4)$, risk of developing SUD $(n=3)$, screening and diagnosis $(n=7)$, psychosocial treatment $(n=5)$, pharmacological treatment $(n=11)$, and complementary treatments ( $n=$ 7). Routine screening is recommended for ADHD in adolescent patients in substance abuse treatment and for SUD in adolescent patients with ADHD in mental healthcare settings. Long-acting stimulants are recommended as the firstline treatment of $A D H D$ in adolescents with concurrent ADHD and SUD, and pharmacotherapy should preferably be embedded in psychosocial treatment. The only remaining no-consensus statement concerned the requirement of abstinence before starting pharmacological treatment in adolescents with ADHD and concurrent SUD. In contrast to the majority, some experts required full abstinence before starting any pharmacological treatment, some were against the use of stimulants in the treatment of these patients (independent of abstinence), while some were against the alternative use of bupropion. Conclusion: This international consensus statement can be used by clinicians and patients together in a shared decision-making process to select the best interventions and to reach optimal outcomes in adolescent patients with concurrent ADHD and SUD.

(c) 2020 S. Karger AG, Basel

\section{Introduction}

Attention-deficit/hyperactivity disorder (ADHD) and substance use disorder (SUD) often co-occur, in both adults and adolescents seeking treatment [1]. The co-occurrence of ADHD and SUD complicates screening, diagnosis, and treatment and is associated with poor treatment outcomes (e.g., [2]). Childhood ADHD is a prominent risk factor for substance misuse and the development of SUD in adolescence and early adulthood, even when the most common comorbidities during childhood - conduct disorder and oppositional defiant disorder - are accounted for (e.g., $[3,4])$. Some studies suggest that stimulant treatment of children with ADHD has a protective effect on the development of SUD in adolescence and early adulthood (e.g., $[5,6]$ ), but other studies did not find such a protective effect (e.g., $[7,8])$.

Consensus Statement on ADHD and SUD in Adolescents
Existing guidelines on ADHD pay little attention to adolescents with comorbid SUD as a distinctive subgroup. Most guidelines only provide some general advice to screen adolescents with ADHD for substance misuse and SUD, recommend to use medications with little or no misuse potential, and/or mention that clinicians should be alert for signs of misuse or diversion of ADHD medication in this group (Guidelines of the Netherlands [9], Australia [10], Scotland [11], Germany [12], Canada [13], the USA [14], and the UK [15]). Moreover, most studies on treatment efficacy of ADHD in youth were conducted in mixed samples of children and adolescents (5-18 years) without separate reporting on the adolescent subgroup (12-18 years). This is unfortunate, because compared with children and adults with ADHD, adolescents with ADHD experience different challenges and treatment demands $[16,17]$.

Early detection of comorbid ADHD among adolescents receiving SUD treatment and detection of SUD and age-inappropriate use of substances among adolescents receiving ADHD treatment is of vital importance for successful treatment of these comorbid disorders. Although reliable and valid screening instruments and structured diagnostic interviews are available for both disorders [1821], systematic screening for ADHD in SUD treatment settings and, conversely, for SUD in psychiatric settings is not routine in clinical practice. Consequently, comorbid ADHD and SUD often remains undetected and hence untreated.

Evidence on the psychosocial and pharmacological treatment of comorbid ADHD and SUD in adolescents is limited. There are currently no meta-analyses or randomized controlled trials (RCTs) that have investigated the efficacy of psychosocial treatments or complementary interventions in adolescents with concurrent ADHD and SUD. However, there are 11 RCTs that examined psychosocial interventions in adolescents with ADHD but without SUD comorbidity, either in a school setting [22-26] or in a clinical setting [27-32]. A few of these studies provide some indication that adolescents with ADHD may benefit from psychosocial treatment; however, methodological quality of the studies was generally low and treatment types, treatment components, and treatment settings were heterogeneous. Hence, there is no robust evidence to recommend specific psychosocial treatments for ADHD in adolescents with or without comorbid SUD. Recently, the first trial comparing cognitive behavioral therapy (CBT) for SUD with CBT targeted at both ADHD and SUD in adults with concurrent SUD and ADHD showed a favorable add-on effect of this integrated CBT

Eur Addict Res 2020;26:223-232 225 
over CBT for SUD only on ADHD symptoms but not on substance use [33]. Taken together, these findings suggest that CBT may be an effective treatment not only for SUD but also for comorbid ADHD in SUD patients.

Five RCTs have been conducted to study the effect of complementary treatments in adolescents with ADHD but without SUD: cognitive training [26, 34], neurofeedback [35], dietary supplementation with omega-3/6 fatty acids [36], and physical exercise [37]. None yielded robust evidence that these complementary interventions reduce ADHD symptoms in these patients. It is, therefore, unlikely that these interventions will be effective in ADHD adolescents with SUD.

To examine the efficacy of pharmacotherapy in patients with ADHD and concurrent SUD, Cunill et al. [38] conducted a meta-analysis of $13 \mathrm{RCTs}$ and reported mixed results: pharmacotherapy had a (small) beneficial effect on ADHD symptoms, but no effect on substance use or abstinence. Unfortunately, in this meta-analysis, no distinction was made between adult and adolescent patients, and no attention was paid to the heterogeneity within and between samples, to the methodological difference between studies or to variations in dosing. In their systematic review of ADHD pharmacotherapy trials in SUD patients, Carpentier and Levin [39] provide some possible explanations for the mostly negative study findings, including the high prevalence of polydrug use in most study samples, the presence of psychiatric comorbidity in addition to ADHD and SUD, the possibility of suboptimal dosing of stimulant medication, the generally low treatment retention in the trials, and the influence of concurrent treatment, mostly CBT.

To date, only 4 (placebo-)controlled studies have been conducted on the efficacy of pharmacotherapy in adolescents with concurrent ADHD and SUD with a total of less than 500 patients: 2 trials with long-acting methylphenidate [40,41], 1 trial with pemoline [42], and 1 trial with atomoxetine [43] as the active compound. None of these studies showed a robust treatment effect on either ADHD or SUD. One study found an effect on ADHD, but included only 16 patients [41]; one study reported an effect on ADHD, but with contradictory findings between the primary and secondary ADHD outcome measures [42]; one study reported an effect on ADHD and SUD, but with contradictory findings between the primary and secondary ADHD outcomes and between the substance use selfreport and urinalysis data [40]; and one study found no effect on ADHD and SUD on any of the outcome measures [43]. In contrast, virtually, all trials of ADHD pharmacotherapy in adolescents with ADHD but without
SUD comorbidity showed a moderate-to-large effect of the medication on ADHD symptoms, including studies with long-acting methylphenidate [44-47], lisdexamfetamine [48], extended-release mixed amphetamine salts [49], pemoline [50], atomoxetine [51], and long-acting guanfacine [52-54] as the active compound.

Pharmacological treatments in adolescents with concurrent ADHD and SUD were well tolerated in all 4 studies [40-43]. Adverse events were generally more prevalent in adolescents in the active medication groups, but these were mostly mild and transient. Reported studyrelated serious adverse events (SAEs) in the active medication groups were absent or rare $(\leq 1 \mathrm{SAE})$ in all studies, with no excess of SAEs in any of the active medication groups, compared with placebo. Negative interactions between study medication and the adolescent's substance use were only investigated in the trial of Riggs et al. [40] and were reported only by $2.8 \%$ of the patients receiving methylphenidate. There was no indication that stimulant medication resulted in deterioration of SUD.

While generally well tolerated, stimulant medications and atomoxetine have been associated with a range of short- and long-term cardiovascular adverse effects. Childress [55] reviewed the extended-release stimulants [56] for treating ADHD and found an overall small increase in blood pressure and heart rate for both amphetamines and methylphenidate. In a recent, comprehensive meta-analysis of RCTs on this subject, Liang et al. [56] found that both methylphenidate and atomoxetine in children and adolescents were associated with increased heart rate and systolic blood pressure but were not associated with the number of adverse cardiac events, compared with placebo. Earlier large-scale registry studies have found no evidence that ADHD medications, including stimulants, were associated with increased risk of serious cardiovascular events in children and young adults [57] or in young and middle-aged adults [58].

In conclusion, research on the diagnosis and treatment of adolescents with ADHD and SUD is scarce, and the evidence from the few available studies in this subgroup is not strong enough to justify robust treatment recommendations. Therefore, as part of the initiative of the International Collaboration on ADHD and Substance Abuse (ICASA), we performed a modified Delphi study based on scientific and clinical evidence with a large multidisciplinary international group of experts who were asked to rate a set of statements regarding (a) the effect of ADHD treatment of children on the development of SUD in adolescence and early adulthood, and (b) the possibilities for screening, diagnosis, and treatment of adoles- 
cent patients with co-occurring ADHD and SUD. ICASA previously initiated the development of a comparable consensus document on the screening, diagnosis, and treatment of adult patients with concurrent ADHD and SUD [59].

\section{Methods}

We conducted a modified Delphi process [60] with the following steps:

1. A systematic literature review was performed by 3 members of the expert group (V.H., H.Ö., and R.S.) [61] on (a) the effect of ADHD treatment of children on the development of SUD in adolescence and (b) the possibilities for screening, diagnosis, and treatment of adolescent patients with co-occurring ADHD and SUD [61].

2. Based on this systematic review, initial statements for a consensus document were selected by the authors of the review (V.H., H.Ö., and R.S.) and 4 members of ICASA: W.v.d.B., G.v.d.G., A.S., M.N., and M.H.

3. A multidisciplinary international group of 62 experts was invited for the consensus process: 44 ICASA members (71\%) and 18 child and adolescent ADHD-SUD experts nominated by these ICASA members (29\%). Of these 62 invited experts, 55 participated in the Delphi process: 37 (67\%) ICASA-members and $18(33 \%)$ invited child and adolescent experts. The 55 participating experts (including the 8 experts involved in the selection of the statements) were from 17 countries and 5 continents (Europe, North America, Australia, Asia, and Africa) and included 15 (child and adolescent) psychiatrists, 14 (child and adolescent) psychologists, 16 addiction physicians, 6 scientists, 2 pharmacists, 1 mental health nurse, and 1 epidemiologist.

4. All statements (resulting from step 2) and the systematic review (resulting from step 1) were sent to the group of 55 experts. They were asked to (a) rate all statements on a scale of $1-5(1=$ most disagree, 2 = disagree, $3=$ neutral, $4=$ agree, and $5=$ strongly agree) based on their knowledge, clinical experience, and - if desired - the systematic review, (b) provide comments on the content and/or the phrasing of the statements if they felt that this was needed or useful, and (c) propose any additional statements they felt would be useful. Consensus between experts for each statement was defined a priori as at least $95 \%$ of all the ratings being greater than or equal to 3 (strongly agree, agree, and neutral), and thus, no more than 2 experts with a rating on a specific statement equal to or smaller than 2 (disagree and most disagree). Although arbitrary, we considered a "neutral" rating in our dichotomous consensus measure to reflect (some) agreement rather than disagreement, because the rater at least did not disagree with the statement. The same procedure was used in a recent consensus article on baclofen as a viable treatment for alcohol dependence [62].

5. After all ratings and comments were received and consensus was calculated, all statements without consensus, statements with consensus but useful comments that resulted in a different phrasing, and additional statements proposed by the experts were sent out for a second round of rating by all experts.

Consensus Statement on ADHD and SUD

in Adolescents
6. Based on this second round of ratings, consensus was calculated for this subgroup of statements using the same a priori defined rules. Statements that still did not reach expert consensus were sent out to all experts for a final rating and a final request for comments. In addition, all experts received the full text of the international consensus statement (including abstract, introduction, methods, and discussion/conclusion) with a request to comment on the text.

7. Based on the final round of ratings and comments, the text of the paper was finalized by the senior authors of the current article (H.Ö., R.S., W.v.d.B., and V.H.) and submitted for publication.

\section{Results}

Of the 55 experts in the consensus group, 52 (95\%) participated in the first round, $55(100 \%)$ participated in the second round, and again $55(100 \%)$ participated in the third round of ratings and comments on statements and on the text of the consensus document.

Based on our a priori definition of consensus, 10 of the 36 selected initial statements did not reach consensus in the first round (Table 1). These were adapted and were sent out again for the second round of rating and commenting. In addition, we identified 3 statements with consensus, but with comments that stimulated us to slightly rephrase these statements. These adapted statements were also sent out for the second round of rating and commenting. Finally, some experts proposed additional statements, and of these, we added 1 statement (Table 1, Statement 30) that was also sent out for the second round of rating and commenting. Thus, the second round of rating and commenting involved 14 of the $37(36+1)$ statements.

In the second round of ratings and comments, 11 of the 14 (adapted) statements reached consensus. The remaining 3 statements were further adapted and sent out for a third round of rating and commenting, together with the draft text of the consensus document and a request to provide comments.

In the third round of rating and commenting, 2 of the 3 (adapted) statements reached consensus. Hence, consensus was reached on 36 of the 37 statements. The statement without consensus (Table 1, Statement 22) concerned the requirement of abstinence before starting stimulant pharmacotherapy in adolescents with ADHD and concurrent SUD. In contrast to the majority of experts, some experts required full abstinence before starting any pharmacological treatment, some were against the use of any stimulant in the treatment of these patients (independent of abstinence), and some experts were against the alternative use of bupropion. 
Table 1. Statements on screening, diagnosis, and treatment of adolescents with concurrent ADHD and SUD

Statements

Consensus reached

in round No.

General statements

1 The treatment of concurrent ADHD and SUD in adolescents has received little attention in research and in guidelines. The evidence-base pertaining to the pharmacological and non-pharmacological treatment of this group is very small, and the few trials that have been conducted provide insufficient evidence for strong treatment recommendations. The recommendations in this consensus statement are, therefore, mainly based on a combination of clinical practice and the available trials conducted in adolescents with ADHD without SUD comorbidity and/or adults with ADHD and SUD

2 In adolescents with concurrent ADHD and SUD, treatment of ADHD usually does not result in reduced substance use, and neither does treatment of SUD generally have a positive impact on ADHD symptoms. It is, therefore, recommended that treatment should focus on both disorders concurrently, should pay attention to their interrelationship, and should follow the guidelines for each separate disorder and the general guidelines about treatment of comorbid patients

3 In most cases of concurrent ADHD and SUD in adolescents, it is advisable to start treatment aimed at abstaining from or reducing/ stabilizing the use of substances first, since current SUD may complicate diagnosis and treatment of ADHD. However, start of treatment of ADHD should not unnecessarily be delayed

4 In all cases, it is sensible to ask adolescents with ADHD and SUD whether and how they would like to involve their parents and/or other confidants in the treatment

Risk of developing SUD

5 Childhood ADHD is a serious risk factor for developing SUD in adolescence, especially when ADHD occurs in combination with conduct disorder or oppositional defiant disorder

6 Data from the available scientific studies strongly suggest that stimulant treatment of childhood ADHD does not increase the risk of developing SUD in adolescence

7 Data from the available scientific studies indicate that stimulant treatment of childhood ADHD may reduce the risk of developing SUD in adolescence. The overall effect size of the reduced risk is probably small, but some studies suggest that an earlier start of stimulant treatment with adequate doses is associated with a larger preventive effect

Screening and diagnosis of ADHD and SUD

8 Heavy substance use predicts worse treatment outcomes for both ADHD and SUD. Early detection of these disorders and their comorbidity plays a crucial role in prevention and in the treatment of both disorders in psychiatric and addiction treatment settings

9 Given the high rate of concurrent ADHD and SUD, routine screening for at-risk use of substances and SUD is recommended in adolescents with ADHD entering primary care and mental health treatment settings. Clinicians are advised to explain the confidentiality of the information, and to assess and evaluate the adolescent's substance use in an open and non-judgmental way, preferably with the adolescent alone, without the parent(s) being present

10 Vice versa, routine screening for ADHD is recommended in adolescents entering substance abuse treatment settings

11 It is recommended that screening and diagnostic assessment take place when the patient's substance use is sufficiently stabilized. Only in case of acute intoxication or severe withdrawal symptoms should these assessments be postponed to a later date

12 When diagnosing concurrent ADHD and SUD, the clinician should follow the diagnostic procedures for each separate disorder and should pay special attention to the potential overlap, interactions, and chronological order of symptoms

13 The diagnostic evaluation of ADHD and SUD should be considered as a dynamic process, given that both disorders and their interaction may fluctuate over time

14 The diagnosis of concurrent ADHD and SUD in adolescents should be based on a thorough diagnostic assessment by a trained professional (e.g., child and adolescent psychiatrist, psychiatrist, pediatrician, clinical psychologist, and addiction medicine physician). The use of standardized structured diagnostic instruments is recommended

Psychosocial treatment of comorbid ADHD and SUD

15 Concurrent ADHD and SUD in adolescence is a severe condition and although environmental adaptations (e.g., at home or school) are important in the management of ADHD, psychosocial and/or pharmacological treatment of this comorbid condition should start as soon as possible

16 Psychological treatment in adolescents with comorbid ADHD and SUD should include psychoeducation and motivational interviewing to enhance treatment engagement and retention and CBT for either SUD or both conditions 
Table 1 (continued)

Statements

Consensus reached

in round No.

Pharmacological treatment of comorbid ADHD and SUD

20 Despite the lack of evidence for the efficacy of pharmacotherapy in adolescents with concurrent ADHD and SUD, it is

1

recommended that pharmacological treatment of ADHD, particularly with psychostimulants, should be considered for this group

21 Each adolescent with concurrent ADHD and SUD and his/her parents should receive information about the option of pharmacotherapy for ADHD and its preconditions and monitoring

22 Before starting stimulant pharmacotherapy in adolescents with concurrent ADHD and SUD, it is important that the adolescents are abstinent or have reduced/stabilized their substance use. If this is not the case, the clinician should consider non-stimulant pharmacotherapy (e.g., atomoxetine, guanfacine, or bupropion)

23 Before starting psychostimulant treatment, the clinician should communicate that treatment will only be continued if it has a demonstrably favorable effect in terms of reduced ADHD symptoms and/or improved functioning

24 Pharmacological treatment in adolescents with concurrent ADHD and SUD should preferably be embedded in psychosocial treatment

25 If the clinician suspects psychostimulant medication misuse or diversion, this is an urgent reason to discuss, and if necessary, terminate psychostimulant treatment and consider non-stimulant treatment. To minimize the risk of misuse and diversion of stimulant medication, it is best to prescribe long-acting instead of short-acting psychostimulants, to avoid long-term and repeat prescriptions and to carefully monitor progress and possible problems

26 Pharmacological treatment of ADHD requires careful titration and monitoring of its effect and possible adverse effects. Higher doses of psychostimulants may be required in patients with ADHD and concurrent SUD than in those without SUD for a favorable effect on both the ADHD symptoms and reduction of substance use

27 First-line pharmacotherapy of ADHD in adolescents with concurrent ADHD and SUD consists of long-acting psychostimulants (e.g., methylphenidate, lisdexamfetamine, dexamphetamine, and mixed amphetamine salts). As second-line pharmacological treatments atomoxetine, guanfacine XR or bupropion can be considered

28 Although comparable at the population level, the efficacy and tolerability of long-acting methylphenidate, (lis)dexamphetamine and extended-release mixed amphetamine salts may differ between individuals. It is, therefore, recommended to test the effect with one of these stimulant medications in a patient with concurrent ADHD and SUD, and, in case of nonresponse at an adequate dose, switch to the next stimulant medication

29 An electrocardiogram is needed before initiating psychostimulant treatment only in adolescents with ADHD and SUD who have a (family) history, symptoms or signs of cardiac disease, and/or who use a medication or illicit drug (e.g., cocaine and amphetamine) that may increase cardiac risk. Heart rate and (systolic) blood pressure should be monitored throughout the course of pharmacological ADHD treatment in all adolescents with concurrent ADHD and SUD

30 In adolescents with ADHD and SUD treated with psychostimulants or atomoxetine, growth and weight should be monitored

Complementary treatment

31 As a "common sense" recommendation, it is wise to discuss the benefits of a healthy lifestyle (balanced diet, good nutrition, regular exercise, scheduled bed and wakening hours, etc.) with the adolescent

32 In adolescents with concurrent ADHD and SUD, no studies have been conducted that provide convincing evidence for a beneficial effect of computerized cognitive training programs on ADHD symptoms or related functional impairment. Computerized cognitive training programs are, therefore, not recommended

33 In adolescents with concurrent ADHD and SUD, no studies have been conducted that provide convincing evidence for a beneficial effect of (EEG) neurofeedback on ADHD symptoms or related functional impairment. (EEG) Neurofeedback is, therefore, not recommended

34 In adolescents with concurrent ADHD and SUD, no studies have been conducted that provide convincing evidence for a beneficial effect of dietary interventions (e.g., restrictive or elimination diets) on ADHD symptoms or related functional impairment. Dietary interventions are, therefore, not recommended

35 In adolescents with concurrent ADHD and SUD, no studies have been conducted that provide convincing evidence for a beneficial effect of meditation/mindfulness-based therapies on ADHD symptoms or related functional impairment. Meditation/mindfulnessbased therapies are, therefore, not recommended as primary treatment but may be used as an add-on intervention in some patients

36 In adolescents with concurrent ADHD and SUD, no studies have been conducted that provide convincing evidence for a beneficial effect of physical exercise interventions on ADHD symptoms or related functional impairment. Physical exercise interventions are, therefore, not recommended as primary treatment but may be used as add-on interventions in some patients

37 In adolescents with concurrent ADHD and SUD, no studies have been conducted that provide convincing evidence for a beneficial effect of traditional and/or herbal medicine on ADHD symptoms or related functional impairment. Traditional and/or herbal medicine are, therefore, not recommended

Agreement with the statement was rated on a 5 -point scale $(1=$ most disagree, $2=$ disagree, $3=$ neutral, $4=$ agree, and $5=$ strongly agree $)$. In each of the 3 rounds, consensus for a statement was reached if at least 95\% of the experts provided a score of 3 or higher. ADHD, attention-deficit/hyperactivity disorder; SUD, substance use disorder; CBT, cognitive behavioral treatment. 
In summary, consensus was reached on 23 of the 36 statements in round $1(64 \%)$ and - after adaptation of the remaining 13 statements plus 1 new statement - on 11 of the 14 statements in round 2 (overall consensus $23+11$ of 37 statements $=34 / 37=92 \%)$. After adaptation of the remaining 3 statements, consensus in round 3 was reached on 2 of the 3 statements, resulting in an overall consensus about 36 of the 37 statements (97\%).

\section{Discussion and Conclusion}

The effect of treatment of childhood ADHD on the development of adolescent SUD and the screening, diagnosis, and treatment of concurrent ADHD and SUD in adolescents has received little attention in research and in treatment guidelines. The evidence-base on these issues is limited and not robust enough for strong clinical recommendations. At the same time, adolescent patients with ADHD and concurrent SUD are in serious need of treatment to improve clinical and psychosocial outcomes and to prevent chronicity. In an attempt to fill this need, we performed a modified Delphi study with the aim of obtaining a set of consensus statements pertaining to these issues.

The study shows that a multidisciplinary international group of experts was able to reach a high level of consensus on 36 of 37 statements about the treatment of childhood ADHD to prevent the development of SUD later in life and about the screening, diagnosis, and treatment of adolescents with co-occurring ADHD and SUD. Consensus was easily reached in the first round for $64 \%$ of the statements and - after adaptations of the original statements - this percentage mounted to $92 \%$ in the second and $97 \%$ in the third round. The only statement with no consensus concerned the requirement of abstinence or reduced/stabilized substance use before prescribing psychostimulants to adolescents with concurrent ADHD and SUD. The reasons for this lack of consensus were multiple. Some experts preferred to minimize the potential risks by demanding strict abstinence as a precondition for pharmacotherapy, while others were worried that a strict abstinence policy would exclude a large group of patients from receiving effective treatment. Some other experts were against the use of psychostimulants in these patients altogether (i.e., independent of whether patients were abstinent at the start of such a treatment) mainly because of the risk of abuse and/or diversion. Finally, some experts were strongly opposed to the use of bupropion as an alternative to psy- chostimulants in non-abstinent patients, mainly due to a lack of evidence of its effect and the risk of bupropioninduced seizures. It is important to note that the existing national treatment guidelines on treating ADHD in children and adolescents show similar disagreements on these issues.

The current study has both strengths and limitations. The main strengths are (1) the presence of a systematic review as a shared knowledge base for all participating experts, (2) a sizable group of experts from different countries with different treatment cultures, and (3) a very high initial and final response rate. The main limitations are (1) possible bias in the selection of the experts, given that most participating scientists and clinicians had a special interest in the topic of ADHD and SUD, child and adolescent experts were recruited through co-optation by the ICASA Network, and pediatricians and patient representatives were absent in the expert group, and (2) the lack of an official approval of our consensus statement by scientific, professional, and patient organizations. In order to (partly) remedy the latter limitation, we will circulate the results of this consensus article directly to the international scientific, professional, and patient organizations in the mental health and addiction field. In addition, we will present the results at national and international conferences.

The current set of consensus statements can be used by clinicians and patients together in a shared decisionmaking process to select the most appropriate treatments and to reach optimal outcomes for adolescents with complex problems based on the integration of scientific knowledge, clinical experience, and patient preference. Finally, we would like to emphasize that our consensus statement on concurrent ADHD and SUD is not a replacement for an evidence-based guideline and that more high-quality studies, including both RCTs and long-term naturalistic follow-up studies are needed [63].

\section{Acknowledgements}

We gratefully acknowledge the ICASA network for initiating the research of this study.

\section{Statement of Ethics}

The authors have no ethical conflicts to disclose. 


\section{Disclosure Statement}

The authors have no conflicts of interest to declare.

\section{Funding Sources}

The research was supported by a financial contribution of ICASA to the Parnassia Addiction Research Centre (PARC) (Dr. Özgen, Dr. Spijkerman, Dr. Hendriks).

\section{Author Contributions}

V.H., H.Ö., and R.S. conducted the systematic review that informed the development of this consensus statement. H.Ö., R.S., M.N., M.H., A.S., G.v.d.G., W.v.d.B., and V.H. were responsible for the selection of the initial statements for this consensus document and drafted the initial document and its revisions. The senior authors H.Ö., R.S., W.v.d.B., and V.H. were responsible for collecting and coordinating the feedback during each consensus round and for finalizing the text of the paper. All authors contributed to the manuscript and approved its final version.

\section{References}

1 van Emmerik-van Oortmerssen $\mathrm{K}$, van de Glind G, van den Brink W, Smit F, Crunelle $\mathrm{CL}$, Swets M, et al. Prevalence of attention-deficit hyperactivity disorder in substance use disorder patients: a meta-analysis and meta-regression analysis. Drug Alcohol Depend. 2012 Apr;122(1-2):11-9.

2 Wilens TE, Morrison NR. Substance-use disorders in adolescents and adults with ADHD: focus on treatment. Neuropsychiatry. 2012 Aug;2(4):301-12.

3 Groenman AP, Oosterlaan J, Rommelse N, Franke B, Roeyers H, Oades RD, et al. Substance use disorders in adolescents with attention deficit hyperactivity disorder: a 4-year follow-up study. Addiction. 2013 Aug;108(8): 1503-11.

4 Groenman AP, Janssen TWP, Oosterlaan J. Childhood psychiatric disorders as risk factor for subsequent substance abuse: a meta-analysis. J Am Acad Child Adolesc Psychiatry. 2017 Jul;56(7):556-69.

5 Groenman AP, Schweren LJS, Weeda W, Luman M, Noordermeer SDS, Heslenfeld DJ, et al. Stimulant treatment profiles predicting $\mathrm{CO}^{-}$ occurring substance use disorders in individuals with attention-deficit/hyperactivity disorder. Eur Child Adolesc Psychiatry. 2019 Sep 1; 28(9):1213-22.

6 Quinn PD, Chang Z, Hur K, Gibbons RD, Lahey BB, Rickert ME, et al. ADHD medication and substance-related problems. Am J Psychiatry. 2017 Sep 1;174(9):877-85.

7 Humphreys KL, Eng T, Lee SS. Stimulant medication and substance use outcomes: a metaanalysis. JAMA Psychiatry. 2013 Jul 1;70(7): 740-9.

8 Molina BS, Hinshaw SP, Eugene Arnold L, Swanson JM, Pelham WE, Hechtman L, et al. Adolescent substance use in the multimodal treatment study of attention-deficit/hyperactivity disorder (ADHD) (MTA) as a function of childhood ADHD, random assignment to childhood treatments, and subsequent medication. J Am Acad Child Adolesc Psychiatry. 2013 Mar 1;52(3):250-63.

9 Landelijke Stuurgroep Multidisciplinaire richtlijnontwikkeling in de GGZ. Multidisciplinaire Richtlijn ADHD bij Kinderen en Jeugdigen. Utrecht: Trimbos-Instituut; 2005.

10 The Royal Australasian College of Physicians (RACP). Draft Australian Guidelines on Attention Deficit Hyperactivity Disorder
(ADHD). 2009. Available from: https://www. racp.edu.au/docs/default-source/advocacylibrary/pa-australian-guidelines-on-adhddraft.pdf?sfvrsn=c905321a $\_$.

11 Scottish Intercollegiate Guidelines Network Edinburgh: Scottish Intercollegiate Guidelines Network (SIGN); Management of attention deficit and hyperkinetic disorders in children and young people: a national clinical guideline [Internet]; 2009 Oct. Available from: http:// www.sign.ac.uk/.

12 Association of the Scientific Medical Societies in Germany (AWMF) online. Interdisciplinary Evidence- and Consensus-Based Guideline "Attention Deficit/Hyperactivity Disorder in Children, Young People and Adults". [in German]; 2018. https://www.awmf.org/uploads/tx_szleitlinien/028-0451_S3_ADHS_2018-06.pdf.

13 Canadian ADHD Resource Alliance (CADDRA). Canadian ADHD practice guidelines. 4th ed. Toronto, ON: CADDRA; 2018.

14 Wolraich ML, Hagan JF, Wolraich M, Brown L, Brown RT, DuPaul G, et al. ADHD: clinical practice guideline for the diagnosis, evaluation, and treatment of attention-deficit/hyperactivity disorder in children and adolescents. Pediatrics. 2019 Oct 1;128(5):1007-22.

15 National Institute for Health Care Excellence (NICE). Attention defificit hyperactivity disorder: diagnosis and management. NG87; March 2018. Available from: www.nice.org.uk/guidance/ng87.

16 Chan E, Fogler JM, Hammerness PG. Treatment of attention-deficit/hyperactivity disorder in adolescents: a systematic review. JAMA. 2016 May 10;315(18):1997-2008.

17 Sibley MH, Kuriyan AB, Evans SW, Waxmonsky JG, Smith BH. Pharmacological and psychosocial treatments for adolescents with ADHD: an updated systematic review of the literature. Clin Psychol Rev. 2014 Apr 1;34(3): 218-32.

18 Knight JR, Sherritt L, Harris SK, Gates EC, Chang G. Validity of brief alcohol screening tests among adolescents: a comparison of the AUDIT, POSIT, CAGE, and CRAFFT. Alcohol Clin Exp Res. 2003 Jan;27(1):67-73.

19 Babor TF, Robaina K. The alcohol use disorders identification test (AUDIT): a review of graded severity algorithms and national adaptations. Int J Alcohol Drug Res. 2016;5(2):17-24.

20 DuPaul GJ, Reid R, Anastopoulos AD, Lambert MC, Watkins MW, Power TJ. Parent and teacher ratings of attention-deficit/hyperactivity disorder symptoms: factor structure and normative data. Psychol Assess. 2016 Feb; 28(2):214-25

21 Ustun B, Adler LA, Rudin C, Faraone SV, Spencer TJ, Berglund P, et al. The world health organization adult attention-deficit/hyperactivity disorder self-report screening scale for DSM-5. JAMA Psychiatry. 2017 May 1;74(5):520-6.

22 Evans SW, Serpell ZN, Schultz BK, Pastor DA. Cumulative benefits of secondary schoolbased treatment of students with attention deficit hyperactivity disorder. School Psych Rev. 2007;36(2):256-73.

23 Evans SW, Langberg JM, Schultz BK, Vaughn A, Altaye M, Marshall SA, et al. Evaluation of a school-based treatment program for young adolescents with ADHD. J Consult Clin Psychol. 2016;84(1):15-30.

24 Langberg JM, Epstein JN, Becker SP, GirioHerrera E, Vaughn AJ. Evaluation of the homework, organization, and planning skills (HOPS) intervention for middle school students with ADHD as implemented by school mental health providers. School Psych Rev. 2012 Sep 1;41(3):342-64.

25 Schramm SA, Hennig T, Linderkamp F. Training problem solving and organizational skills in adolescents with attention-deficit/hyperactivity disorder: a randomized controlled trial. J Cogn Educ Psychol. 2016 Jan 1;15(3):391-411.

26 Steeger CM, Gondoli DM, Gibson BS, Morrissey RA. Combined cognitive and parent training interventions for adolescents with ADHD and their mothers: a randomized controlled trial. Child Neuropsychol. 2016 May 18; 22(4):394-419.

27 Barkley RA, Edwards G, Laneri M, Fletcher K, Metevia L. The efficacy of problem-solving communication training alone, behavior management training alone, and their combination for parent-adolescent conflict in teenagers with ADHD and ODD. J Consult Clin Psychol. 2001 Dec;69(6):926-41.

28 Boyer BE, Geurts HM, Prins PJ, Van der Oord S. Two novel CBTs for adolescents with ADHD: the value of planning skills. Eur Child Adolesc Psychiatry. 2015 Sep 1;24(9):1075-90.

29 Sibley MH, Graziano PA, Kuriyan AB, Coxe S, Pelham WE, Rodriguez L, et al. Parent-teen behavior therapy + motivational interviewing for adolescents with ADHD. J Consult Clin Psychol. 2016 Aug;84(8):699-712. 
30 Sibley MH, Rodriguez L, Coxe S, Page T, Espinal K. Parent-teen group versus dyadic treatment for adolescent adhd: what works for whom? J Clin Child Adolesc Psychol. 2019 Apr 18;16:1-17.

31 Sprich SE, Safren SA, Finkelstein D, Remmert JE, Hammerness P. A randomized controlled trial of cognitive behavioral therapy for ADHD in medication-treated adolescents. J Child Psychol Psychiatry. 2016 Nov;57(11):1218-26.

32 Vidal R, Castells J, Richarte V, Palomar G, García M, Nicolau R, et al. Group therapy for adolescents with attention-deficit/hyperactivity disorder: a randomized controlled trial. J Am Acad Child Adolesc Psychiatry. 2015 Apr 1; 54(4):275-82.

33 van Emmerik-van Oortmerssen K, Vedel E, Kramer FJ, Blankers M, Dekker JJM, van den Brink W, et al. Integrated cognitive behavioral therapy for ADHD in adult substance use disorder patients: results of a randomized clinical trial. Drug Alcohol Depend. 2019 Apr 1; 197:28-36.

34 Gray SA, Chaban P, Martinussen R, Goldberg R, Gotlieb H, Kronitz R, et al. Effects of a computerized working memory training program on working memory, attention, and academics in adolescents with severe $\mathrm{LD}$ and comorbid ADHD: a randomized controlled trial. J Child Psychol Psychiatry. 2012 Dec;53(12):1277-84.

35 Bink M, Bongers IL, Popma A, Janssen TW, van Nieuwenhuizen C. 1-year follow-up of neurofeedback treatment in adolescents with attention-deficit hyperactivity disorder: randomised controlled trial. BJPsych Open. 2016 Mar;2(2):107-15.

36 Matsudaira T, Gow RV, Kelly J, Murphy C, Potts L, Sumich A, et al. Biochemical and psychological effects of omega-3/6 supplements in male adolescents with attention-deficit/hyperactivity disorder: a randomized, placebo-controlled, clinical trial. J Child Adolesc Psychopharmacol. 2015 Dec;25(10):775-82.

37 Ahmed GM, Mohamed S. Effect of regular aerobic exercises on behavioral, cognitive, and psychological response in patients with attention deficit-hyperactivity disorder. Life Sci J. 2011;8(2):366-71.

38 Cunill R, Castells X, Tobias A, Capellà D. Pharmacological treatment of attention deficit hyperactivity disorder with co-morbid drug dependence. J Psychopharmacol. 2015 Jan;29(1): $15-23$.

39 Carpentier PJ, Levin FR. Pharmacological treatment of ADHD in addicted patients: what does the literature tell us? Harv Rev Psychiatr. 2017 Mar/Apr;25(2):50-64.

40 Riggs PD, Winhusen T, Davies RD, Leimberger JD, Mikulich-Gilbertson S, Klein C, et al. Randomized controlled trial of osmotic-release methylphenidate with cognitive-behavioral therapy in adolescents with attention-deficit/hyperactivity disorder and substance use disorders. J Am Acad Child Adolesc Psychiatry. 2011 Sep 1;50(9):903-14.

41 Szobot CM, Rohde LA, Katz B, Ruaro P, Schaefer $\mathrm{T}$, Walcher $\mathrm{M}$, et al. A randomized crossover clinical study showing that methylpheni-
date-SODAS improves attention-deficit/hyperactivity disorder symptoms in adolescents with substance use disorder. Braz J Med Biol Res. 2008 Mar;41(3):250-7.

42 Riggs PD, Hall SK, Mikulich-Gilbertson SK, Lohman M, Kayser A. A randomized controlled trial of pemoline for attention-deficit/ hyperactivity disorder in substance-abusing adolescents. J Am Acad Child Adolesc Psychiatry. $2004 \mathrm{Apr} ; 43(4): 420-9$.

43 Thurstone C, Riggs PD, Salomonsen-Sautel S, Mikulich-Gilbertson SK. Randomized, controlled trial of atomoxetine for attention-deficit/hyperactivity disorder in adolescents with substance use disorder. J Am Acad Child Adolesc Psychiatry. 2010 Jun 1;49(6):573-82.

44 Findling RL, Turnbow J, Burnside J, Melmed R, Civil R, Li Y. A randomized, double-blind, multicenter, parallel-group, placebo-controlled, dose-optimization study of the methylphenidate transdermal system for the treatment of ADHD in adolescents. CNS Spectr. 2010 Jul;15(7):419-30.

45 Newcorn JH, Nagy P, Childress AC, Frick G, Yan B, Pliszka S. Randomized, double-blind, placebo-controlled acute comparator trials of lisdexamfetamine and extended-release methylphenidate in adolescents with attention-deficit/hyperactivity disorder. CNS Drugs. 2017 Nov;31(11):999-1014.

46 Pelham WE, Smith BH, Evans SW, Bukstein O, Gnagy EM, Greiner AR, et al. The effectiveness of short- and long-acting stimulant medications for adolescents with ADHD in a naturalistic secondary school setting. J Atten Disord. 2017;21(1):40-5.

47 Wilens TE, McBurnett K, Bukstein O, McGough J, Greenhill L, Lerner M, et al. Multisite controlled study of OROS methylphenidate in the treatment of adolescents with attentiondeficit/hyperactivity disorder. Arch Pediatr Adolesc Med. 2006 Jan;160(1):82-90.

48 Findling RL, Childress AC, Cutler AJ, Gasior M, Hamdani M, Ferreira-Cornwell MC, et al. Efficacy and safety of lisdexamfetamine dimesylate in adolescents with attention-deficit/hyperactivity disorder. J Am Acad Child Adolesc Psychiatry. 2011 Apr;50(4):395-405.

49 Spencer TJ, Wilens TE, Biederman J, Weisler RH, Read SC, Pratt R. Efficacy and safety of mixed amphetamine salts extended release (adderall XR) in the management of attentiondeficit/hyperactivity disorder in adolescent patients: a 4-week, randomized, double-blind, placebo-controlled, parallel-group study. Clin Ther. 2006 Feb;28(2):266-79.

50 Bostic JQ, Biederman J, Spencer TJ, Wilens TE, Prince JB, Monuteaux MC, et al. Pemoline treatment of adolescents with attention deficit hyperactivity disorder: a short-term controlled trial. J Child Adolesc Psychopharmacol. 2000 Fall;10(3):205-16.

51 Bangs ME, Bangs ME, Emslie GJ, Spencer TJ, Ramsey JL, Carlson C, et al. Efficacy and safety of atomoxetine in adolescents with attentiondeficit/hyperactivity disorder and major depression. J Child Adolesc Psychopharmacol. 2007 Aug;17(4):407-20.
52 Biederman J, Melmed RD, Patel A, McBurnett $\mathrm{K}$, Konow J, Lyne A, et al. A randomized, double-blind, placebo-controlled study of guanfacine extended release in children and adolescents with attention-deficit/hyperactivity disorder. Pediatrics. 2008 Jan;121(1):e73-84.

53 Sallee FR, McGough J, Wigal T, Donahue J, Lyne A, Biederman J, et al. Guanfacine extended release in children and adolescents with attention-deficit/hyperactivity disorder: a placebo-controlled trial. J Am Acad Child Adolesc Psychiatry. 2009 Feb;48(2):155-65.

54 Wilens TE, Robertson B, Sikirica V, Harper L, Young JL, Bloomfield R, et al. A randomized, placebo-controlled trial of guanfacine extended release in adolescents with attention-deficit/ hyperactivity disorder. J Am Acad Child Adolesc Psychiatry. 2015 Nov;54(11):916-e2.

55 Childress A. The safety of extended-release drug formulations for the treatment of ADHD. Expert Opin Drug Saf. 2017 May;16(5):603-15.

56 Liang EF, Lim SZ, Tam WW, Ho CS, Zhang MW, McIntyre RS, et al. The effect of methylphenidate and atomoxetine on heart rate and systolic blood pressure in young people and adults with attention-deficit hyperactivity disorder (ADHD): systematic review, meta-analysis, and meta-regression. Int J Environ Res Public Health. 2018 Aug 20;15(8):1789.

57 Cooper WO, Habel LA, Sox CM, Chan KA, Arbogast PG, Cheetham TC, et al. ADHD drugs and serious cardiovascular events in children and young adults. N Engl J Med. 2011 Nov 17;365(20):1896-904.

58 Habel LA, Cooper WO, Sox CM, Chan KA, Fireman BH, Arbogast PG, et al. ADHD medications and risk of serious cardiovascular events in young and middle-aged adults. JAMA. 2011 Dec 28;306(24):2673-83.

59 Crunelle CL, van den Brink W, Moggi F, Konstenius M, Franck J, Levin FR, et al. International consensus statement on screening, diagnosis and treatment of substance use disorder patients with comorbid attention deficit/hyperactivity disorder. Eur Addict Res. 2018; 24(1):43-51.

60 Jones J, Hunter D. Consensus methods for medical and health services research. BMJ. 1995 Aug 5;311(7001):376-80.

61 Hendriks V, Özgen H, Spijkerman R. Screening, diagnosis and treatment of adolescents with concurrent attention-deficit/hyperactivity disorder: a systematic review. background document to inform the development of a consensus statement. The Hague: Parnassia Addiction Research Centre (PARC), Parnassia Psychiatric Institute, Parnassia Group. Forthcoming 2020.

62 Agabio R, Sinclair JM, Addolorato G, Aubin HJ, Beraha, EM, Caputo F, et al. Baclofen for the treatment of alcohol use disorder: the $\mathrm{Ca}$ gliari statement. Lancet Psychiatry. 2018 Dec; 5(12):957-60.

63 van de Glind G, Brynte C, Skutle A, Kaye S, Konstenius M, Levin F, et al. The international collaboration on ADHD and substance abuse (ICASA): mission, results, and future activities. Eur Addict Res. Forthcoming 2020. 